BMJ Open Diabetes

Research \& Care

\section{Circulating sex hormone binding globulin levels are modified with intensive lifestyle intervention, but their changes did not independently predict diabetes risk in the Diabetes Prevention Program}

To cite: Aroda VR, Christophi CA, Edelstein SL, et al. Circulating sex hormone binding globulin levels are modified with intensive lifestyle intervention, but their changes did not independently predict diabetes risk in the Diabetes Prevention Program. BMJ Open Diab Res Care 2020;8:e001841. doi:10.1136/ bmjdrc-2020-001841

- Supplemental material is published online only. To view please visit the journal online (http://dx.doi.org/10.1136/ bmjdrc-2020-001841).

Received 21 August 2020 Revised 27 October 2020 Accepted 29 0ctober 2020

Check for updates

C Author(s) (or their employer(s)) 2020. Re-use permitted under CC BY-NC. No commercial re-use. See rights and permissions. Published by BMJ.

For numbered affiliations see end of article.

Correspondence to Dr Vanita R Aroda; varoda@bwh.harvard.edu

\section{ABSTRACT}

Introduction Sex hormone binding globulin (SHBG) levels are reported to be inversely associated with diabetes risk. It is unknown whether diabetes prevention interventions increase SHBG and whether resultant changes in SHBG affect diabetes risk. The purpose of this analysis was to determine whether intensive lifestyle intervention (ILS) or metformin changed circulating SHBG and if resultant changes influenced diabetes risk in the Diabetes Prevention Program (DPP).

Research design and methods This is a secondary analysis from the DPP (1996-2001), a randomized trial of ILS or metformin versus placebo on diabetes risk over a mean follow-up of 3.2 years. The DPP was conducted across 27 academic study centers in the USA. Men, premenopausal and postmenopausal women without hormone use in the DPP were evaluated. The DPP included overweight/obese persons with elevated fasting glucose and impaired glucose tolerance. Main outcomes measures were changes in SHBG levels at 1 year and risk of diabetes over 3 years.

Results ILS resulted in significantly higher increases (postmenopausal women: $p<0.01$ ) or smaller decrements (men: $p<0.05$; premenopausal women: $p<0.01$ ) in SHBG compared with placebo or metformin. Changes in SHBG were primarily attributable to changes in adiposity. There were no consistent associations of change in SHBG with the risk of diabetes by treatment arm or participant group. Conclusions Lifestyle intervention may be associated with favorable changes in circulating SHBG, which is largely due to changes in adiposity. Changes in circulating SHBG do not independently predict reductions in diabetes incidence.

\section{INTRODUCTION}

Sex hormone binding globulin (SHBG) levels have been reported to be inversely proportional to diabetes risk in multiple studies. In longitudinal observational studies, for example, higher

\section{Significance of this study}

What is already known about this subject?

- Sex hormone binding globulin (SHBG) levels are inversely associated with diabetes risk. SHBG levels are also inversely associated with insulin resistance and measures of glycemia. Previous short-term randomized trials suggest an effect of interventions, including lifestyle changes, weight loss, and glucose lowering medications, on SHBG levels. It is not known whether diabetes prevention interventions, per se, alter SHBG levels, whether resultant changes in SHBG levels affect diabetes risk and, if so, whether these effects are independent of adiposity.

What are the new findings?

- Randomization to intensive lifestyle intervention in the Diabetes Prevention Program (DPP) modified circulating SHBG levels, whereas metformin did not Specifically, intensive lifestyle intervention increased SHBG levels in postmenopausal women and attenuated the decline in SHBG levels in men and premenopausal women.

- Changes in SHBG levels due to lifestyle intervention were primarily attributable to changes in adiposity.

- Changes in SHBG levels in the DPP did not independently predict reductions in diabetes incidence.

baseline SHBG levels have been prospectively associated with lower risk of incident type 2 diabetes, and conversely lower baseline SHBG levels have been prospectively associated with a higher risk of incident diabetes, even after adjustment of other variables such as age, body mass index, and free sex steroids. ${ }^{1-8}$ Crosssectional studies have likewise demonstrated inverse associations of circulating SHBG levels 


\section{Significance of this study}

How might these results change the focus of research or clinical practice?

- There is interest in understanding the relationship between SHBG levels and diabetes risk, particularly whether SHBG can be independently targeted to reduce diabetes risk or whether the association between SHBG levels and diabetes risk is primarily due to their shared association with adiposity. Our findings suggest that in a higher risk population such as that studied in the DPP, changes in SHBG levels are likely due to changes in adiposity and metabolic factors and that changes in SHBG levels do not independently predict risk of diabetes. Measurement of SHBG does not replace current markers of risk or treatment effect in populations at high risk of type 2 diabetes.

with indices of insulin resistance and with measures of glycemia. $^{9-13}$

Short-term randomized trials have demonstrated an effect of interventions related to glucose homeostasis on circulating SHBG concentrations, including lifestyle changes, weight loss, and medications..$^{914-17}$ No prior publications have evaluated whether changes in circulating SHBG levels as a result of such interventions impact the long-term risk of diabetes. The Diabetes Prevention Program (DPP), a 3-year multicenter randomized controlled clinical trial, compared intensive lifestyle intervention (ILS) with a targeted goal of weight reduction of at least $7 \%$ of initial body weight, metformin, and placebo on the risk of diabetes in overweight/obese individuals with elevated fasting glucose and glucose intolerance. ${ }^{18}$ Earlier reports from the DPP showed that ILS significantly increased SHBG among postmenopausal glucose-intolerant women in the DPP not using exogenous estrogen $(n=382)$, while metformin did not affect endogenous sex hormones or SHBG. ${ }^{19}$ Contrary to expectations, analyses using the complete evaluable DPP population showed that baseline SHBG alone or SHBG single-nucleotide polymorphisms (SNPs) did not predict 3-year incident diabetes in the DPP population. ${ }^{20}$

In the follow-up analyses reported here, we further explore the relationship between circulating SHBG and diabetes risk. Specifically, we evaluated whether interventionassociated changes in SHBG, in turn, impacted diabetes risk in the high-risk DPP population. We did so by: (1) evaluating the extent to which diabetes prevention interventions (ILS, metformin, and placebo) impacted circulating SHBG levels in the DPP population, in subgroups defined by sex and menopausal status; (2) exploring concomitant determinants of SHBG change in the DPP (eg, adiposity, sex hormones, and glucose measures); and (3) examining the effects of change in SHBG on diabetes risk over an average of 3.2-year follow-up.

\section{RESEARCH DESIGN AND METHODS}

Study design

The DPP design, eligibility, and baseline characteristics have been reported previously. ${ }^{1821}$ The DPP enrolled 3234 participants at least 25 years of age, body mass index (BMI) $24 \mathrm{~kg} / \mathrm{m}^{2}$ or higher $\left(\geq 22 \mathrm{~kg} / \mathrm{m}^{2}\right.$ in Asian-Americans), with fasting blood glucose $95-125 \mathrm{mg} / \mathrm{dL}(\leq 125 \mathrm{mg} / \mathrm{dL}$ in American Indians), and impaired glucose tolerance. Participants were randomly assigned to one of three treatments: an intensive lifestyle program (ILS) with a goal of at least $7 \%$ of weight loss through dietary modification and $150 \mathrm{~min} /$ week of moderate intensity exercise, metformin $850 \mathrm{mg}$ twice daily, or matching placebo. Mean follow-up at the end of DPP was 3.2 years. All participants provided written informed consent. The current analyses are from participants who had baseline and 1-year SHBG blood samples and provided approval for the use of their blood samples in secondary analyses. In order to avoid potentially confounding effects of hormone therapy (ie, hormonal contraception or hormone replacement) on SHBG concentrations, users of these medications were excluded from the current analyses.

The final analysis included 2142 men and women with no self-reported hormone use in the DPP who had baseline and year 1 SHBG levels. Participants were classified prior to analysis as: men $(\mathrm{n}=886)$, premenopausal women with no self-reported hormone use $(n=801)$, henceforth referred to as 'premenopausal women', and postmenopausal women with no self-reported hormone use $(n=455)$, referred to as 'postmenopausal women'. Postmenopausal status was defined as age at enrollment over 55 years, or self-reported natural or surgical menopause. Hormone use was assessed by self-reported medications.

\section{Diabetes ascertainment and glucose measures}

Diabetes status was determined by fasting glucose assessment every 6 months and/or by annual $75 \mathrm{~g}$ oral glucose tolerance test. Diabetes was defined as fasting glucose $\geq 126 \mathrm{mg} / \mathrm{dL}$ and/or 2-hour postchallenge glucose $\geq 200 \mathrm{mg} / \mathrm{dL}$, confirmed. $1 /$ fasting insulin was used as a surrogate measure of insulin sensitivity. ${ }^{22}$

\section{SHBG and sex hormone measurements}

SHBG and sex hormone measurements (dehydroepiandrosterone, dehydroepiandrosterone sulfate, estrone; estrone-S, estradiol, testosterone and dihydrotestosterone (DHT)) were conducted on morning fasting plasma samples, without regard to endogenous hormonal cycle. Sex hormones were measured by gas chromatography/ mass spectrometry (Endoceutics, Quebec City, Canada), and SHBG was measured using ELISA (Bioline), as previously described. ${ }^{20} 2324$

\section{Statistical analysis}

Three analysis groups (males, premenopausal women, and postmenopausal women) were considered. Quantitative characteristics with normal distributions were presented as means $\pm \mathrm{SD}$ and compared among groups using analysis of variance. Characteristics that were not normally distributed were presented as median (25th percentile and 75th percentile) and compared across groups using the Kruskal-Wallis test. Qualitative 
characteristics were presented as frequency $(\%)$, and the $\chi^{2}$ test of association was used to compare these characteristics among groups.

The relationship of various characteristics with the change in SHBG from baseline to year 1 was evaluated with multivariable linear regression models. These models were tested separately within each of the different groups of interest, adjusted for treatment and baseline demographics (age, Caucasian race), lifestyle factors (smoking, alcohol intake, and leisure activity), glucose metabolism measures (fasting plasma glucose, 2-hour postchallenge glucose, and 1 /fasting insulin), and adiposity measures (waist circumference and BMI), and sex hormone measures (estradiol, estrone, and testosterone). We identified a number of individually significant factors, which were then evaluated in a set of hierarchical models that were sequentially tested, as: model 1: baseline SHBG, treatment group, demographics, and lifestyle factors; model 2: additionally adjusted for baseline and change in sex hormones; model 3: further adjusted for baseline and change in adiposity; and then model 4: additionally adjusted for baseline and change in glucose parameters.

The association between the change in SHBG and the risk of diabetes was evaluated using Cox proportional hazards regression models, tested separately by treatment group in each of the three participant groups, and adjusted for demographic measures. Correlation analyses were conducted to evaluate the relationship between changes in SHBG and changes in circulating sex hormones and glucose measures. To further evaluate whether changes in SHBG contributed to centrally mediated downstream effects, mediation analyses were performed with change in SHBG as the predictor, change in Follicle-Stimulating Hormone (FSH) as the mediator, and change in circulating sex hormones or change in glucose measures (fasting glucose, hemoglobin Alc (HbA1c), 2-hour postchallenge glucose, homeostatic model assessment of insulin resistance (HOMA-IR), and $1 /$ fasting insulin) as the outcome, after adjusting for treatment, age, and Caucasian race. Statistical analysis was performed with SAS V.9.4, and all tests were two sided done at a significance level of 0.05 .

\section{RESULTS}

\section{Study population}

As described previously, ${ }^{18}$ the original DPP cohort consisted of two-thirds women and $45 \%$ ethnic minorities, with an average age of $51 \pm 11$ years $($ mean \pm SD) at baseline. Baseline descriptive characteristics by groups (men: $\mathrm{n}=886$; premenopausal women: $\mathrm{n}=801$; postmenopausal women: $\mathrm{n}=455$ ) are presented in table 1 . Baseline sex hormones differed as expected between the three groups, with postmenopausal women having lower levels of circulating sex hormones relative to premenopausal women. Baseline SHBG was lowest in postmenopausal women (median (Q1, Q3) of $34.9(25.9,46.2) \mathrm{nmol} / \mathrm{L})$, with a median value of $45.2(30.9,68.6) \mathrm{nmol} / \mathrm{L}$ in premenopausal women and $39.8(26.9,56.3) \mathrm{nmol} / \mathrm{L}$ in men (table 1).

Effect of DPP interventions (ILS, metformin, and placebo) on change in SHBG at 1 year

Figure 1 and online supplemental table 1 show the unadjusted mean of SHBG at baseline and year 1 by treatment arm. In men, SHBG decreased from baseline to year 1 in all three arms, but ILS was associated with the smallest decrement $(-7.91 \pm 22.27 \mathrm{nmol} / \mathrm{L})($ mean $\pm \mathrm{SD})$ compared with metformin $(-12.40 \pm 20.12 \mathrm{nmol} / \mathrm{L})$ or placebo $(-11.97 \pm 17.30 \mathrm{nmol} / \mathrm{L}) \quad(\mathrm{p}<0.01 \mathrm{ILS}$ vs metformin and $\mathrm{p}<0.01$ ILS vs metformin and $\mathrm{p}=0.02$ ILS vs placebo). In premenopausal women, SHBG remained relatively stable in the ILS group $(-0.61 \pm 34.24 \mathrm{nmol} / \mathrm{L})$, whereas it decreased in the metformin $(-10.81 \pm 40.79 \mathrm{nmol} / \mathrm{L})$ and placebo arms $(-9.83 \pm 41.79 \mathrm{nmol} / \mathrm{L}) \quad(\mathrm{p}<0.01 \mathrm{ILS}$ vs metformin, $p<0.01$ ILS vs placebo). Finally, in postmenopausal women, SHBG increased from baseline to 1 year in the ILS group $(+9.19 \pm 17.20 \mathrm{nmol} / \mathrm{L})$, significantly greater than the changes in the metformin $(+0.03 \pm 23.02 \mathrm{nmol} / \mathrm{L})$ or placebo arms $(-0.88 \pm 17.25 \mathrm{nmol} / \mathrm{L}) \quad(\mathrm{p}<0.01$ ILS vs metformin, $\mathrm{p}<0.01$ ILS vs placebo) (figure 1, online supplemental table 1). Overall, changes in SHBG in the ILS participants were significantly different from changes in SHBG in the placebo or metformin groups, with either increases or smaller decrements comparatively.

In a model adjusting for treatment assignment, analysis group (ie, men, premenopausal women, and postmenopausal women), change in SHBG, age at randomization, and race (Caucasian vs not), the interaction of treatment with analysis group was statistically significant $(\mathrm{p}=0.047)$, suggesting the effect of treatment on SHBG was different in the three analysis groups. When tested within each analysis group, in models adjusting for treatment, change in SHBG, age at randomization, and race (Caucasian vs not), the interaction of change in SHBG with treatment arm was statistically significant only in postmenopausal women ( $p=0.023)$, suggesting that in this group the effect of change in SHBG on the risk of diabetes is different among the three treatment arms; in men and premenopausal women, the interaction of change in SHBG with treatment arm was not significant.

\section{Determinants of change in SHBG}

The observed differences between treatment arms suggest a number of hypothesized mediators that might drive the changes in SHBG. Therefore, hierarchical multivariable linear regression models of changes in SHBG from baseline to year 1 were conducted evaluating potential determinants of this change (figure 2, online supplemental table 2). The ILS group continued to have less decrease (men and premenopausal women) or greater increase (postmenopausal women) in SHBG in all groups after adjustment for demographics and lifestyle factors (model 1 ), which were slightly attenuated following additional adjustment for sex hormones (model 2) and completely eliminated following adjustment for adiposity measures 


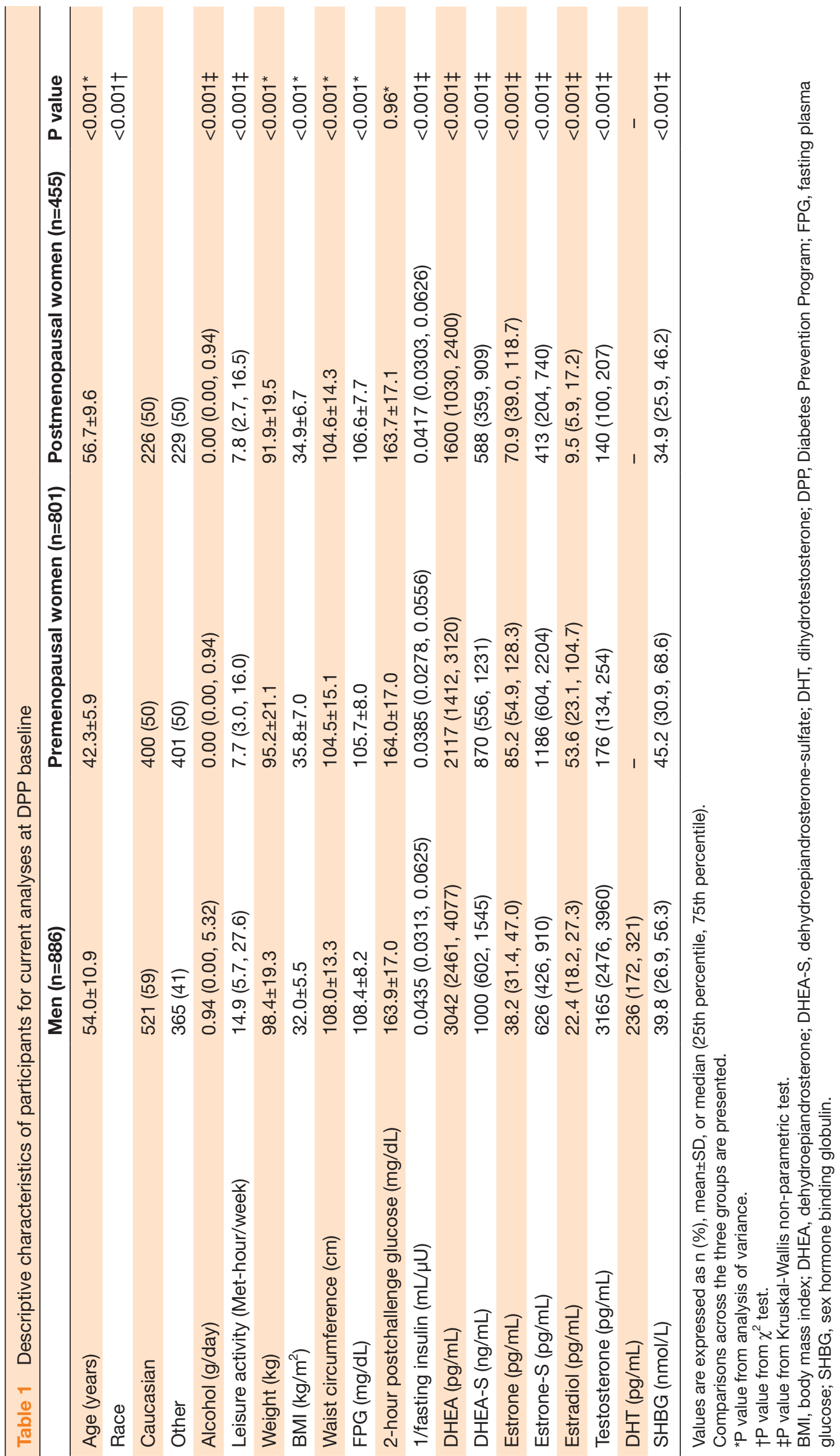


A. Males

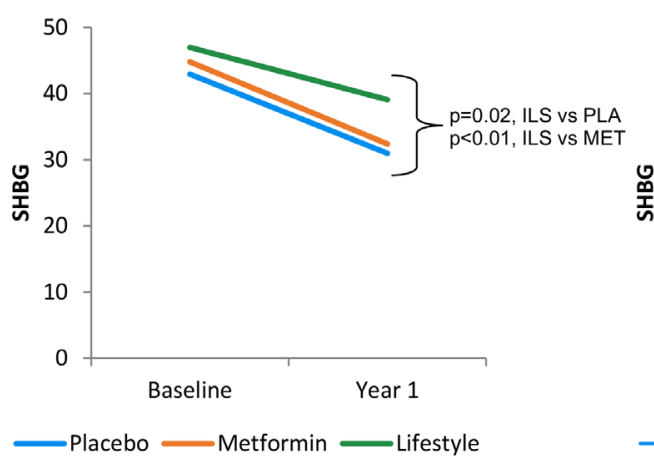

C. Postmenopausal women

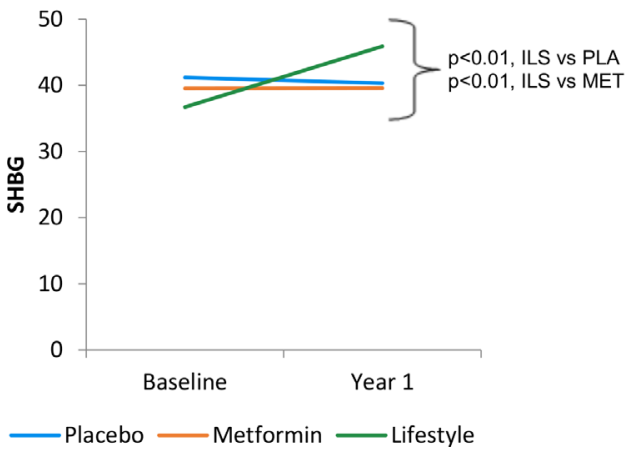

B. Premenopausal women

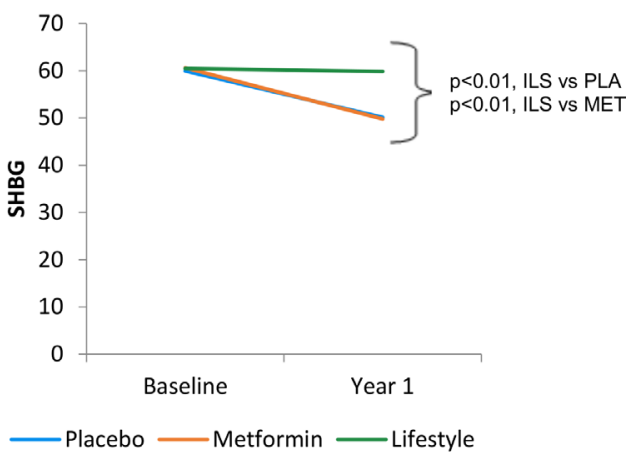

minimally impacted the results (model 4).

\section{Effects of change in SHBG on diabetes risk}

The relationship between 1-year change in SHBG and diabetes risk was inconsistent across treatment arms and participant subgroups (table 2). A significant effect of change in SHBG on diabetes risk was seen in men in the placebo group, HR $0.77,95 \%$ CI 0.63 to $0.95, p=0.01$, for each SD relative increase in SHBG. In addition, a significant effect of change in SHBG on diabetes risk was seen in postmenopausal women in the metformin group (HR $1.61,95 \%$ CI 1.05 to $2.49, \mathrm{p}=0.03$ ). However, these were not consistent findings across treatment arms and participant groups (table 2).

\section{Correlation of SHBG changes with sex hormones and glucose measures}

Changes in SHBG were positively correlated with changes in peripheral sex hormones (change in estradiol and change in testosterone), and inversely correlated with glucose measures (fasting glucose, HbAlc, 2-hour postchallenge glucose), negatively correlated with HOMA-IR and positively correlated with 1 /fasting insulin (table 3 ). These effects were consistent across treatment arms (table 3 ) and were not centrally mediated by FSH (data not shown).

\section{DISCUSSION}

In summary, in the DPP, lifestyle intervention exerted effects to increase or attenuate the decline in SHBG levels, manifested differently within each subgroup. In postmenopausal women, for example, ILS resulted in an increase in SHBG, consistent with prior analyses within the DPP ${ }^{19}$ whereas in men and in premenopausal women in the ILS arm, the decrement in SHBG was less than that seen in the other treatment arms. Metformin had no impact compared with placebo on SHBG in any of the groups. Through multivariable regression models, we found that a large part of the effect of ILS on SHBG levels was attributable to reductions in adiposity, with modest effects of sex hormones, glucose measures, and their changes. Given that low SHBG levels have been reported to be associated with diabetes risk ${ }^{1-7}$ and that low SHBG levels are seen in conditions associated with obesity and insulin resistance (eg, diabetes, polycystic ovary syndrome, and fatty liver disease) ${ }^{9-12} 25-28$ we explored whether the changes in SHBG contributed to reduced diabetes incidence. However, despite these apparently beneficial changes in SHBG, we did not see consistent evidence for SHBG mediating the treatment effects on diabetes prevention. Furthermore, while changes in SHBG were positively correlated with changes in circulating hormones and 1 /fasting insulin, and inversely associated with changes in glucose (fasting and 2-hour postchallenge) and HOMA-IR, mediation analyses did 


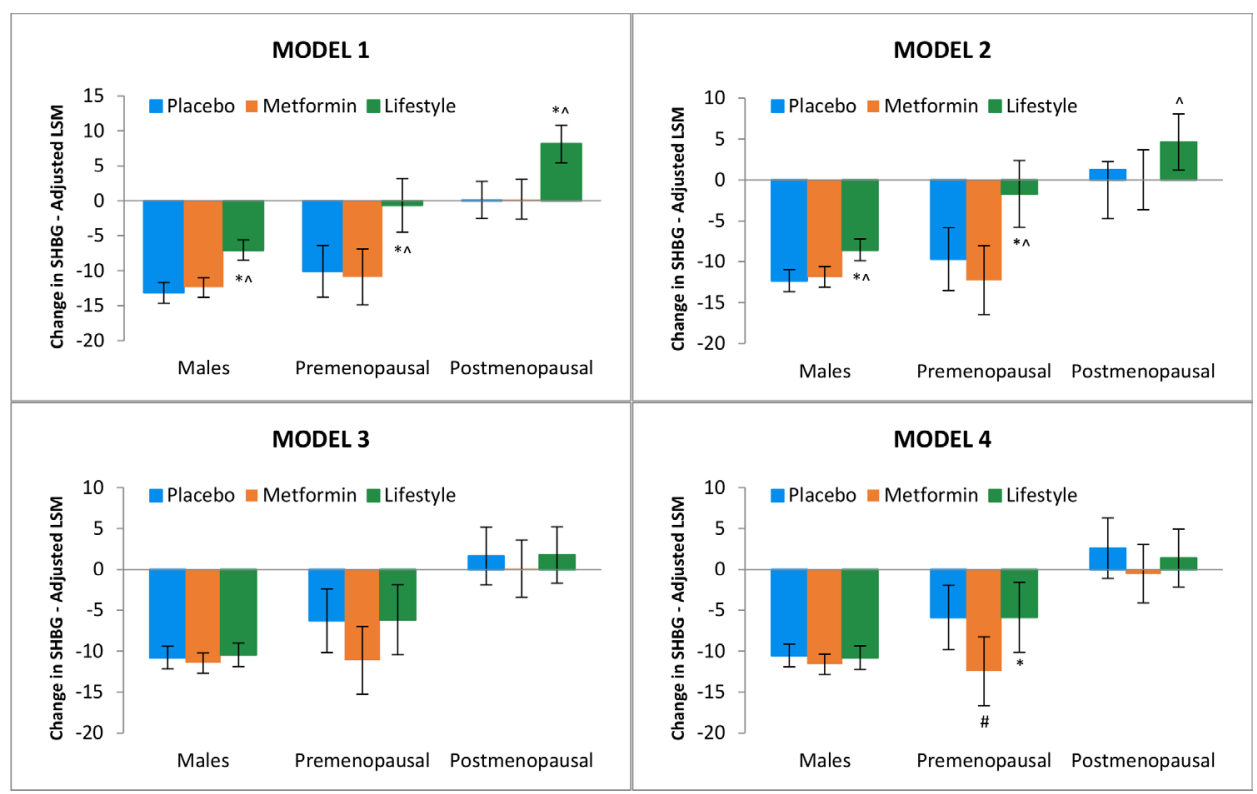

Figure 2 Adjusted mean changes in SHBG from hierarchical modeling of multivariable linear regression models; changes in SHBG from DPP baseline to year 1. Bars represent 95\% Cls. Model 1: change in SHBG, adjusted for age, Caucasian race, smoking, alcohol intake, leisure activity, and baseline SHBG. Model 2: change in SHBG as per model 1, additionally adjusted for sex hormones (estradiol, change in estradiol, estrone, change in estrone, testosterone, and change in testosterone). Model 3: change in SHBG, as per model 2, additionally adjusted for adiposity measures (waist circumference, change in waist circumference, BMI, and change in BMI). Model 4: change in SHBG, as per model 3, additionally adjusted for glucose measures (fasting plasma glucose, change in fasting plasma glucose, 2-hour postchallenge glucose, change in 2-hour postchallenge glucose, 1 /fasting insulin, and change in 1/fasting insulin). *ILS vs. metformin, $p<0.05,{ }^{\wedge} I L S$ vs. placebo, $p<0.05$, \#metformin vs. placebo, p<0.05. BMI, body mass index; DPP, Diabetes Prevention Program; ILS, intensive lifestyle intervention; SHBG, sex hormone binding globulin.

not support a centrally mediated effect of SHBG on these outcomes. Taken together, these observations suggest that changes in SHBG reflect the overall metabolic and hormonal milieu, in particular the effects of weight loss, but are not directly influencing risk of development of diabetes.

Our findings are also consistent with our earlier analyses of baseline SHBG and SHBG SNPs associated with diabetes outcomes and risk of diabetes in the DPP. As earlier reported, while baseline SHBG was cross-sectionally associated with some indicators of insulin resistance and diabetes risk (inverse fasting insulin, insulinogenic index, and waist circumference), SHBG concentration at baseline was not associated with diabetes risk in any of the participant groups evaluated. Furthermore, there was no evident association of the SHBG SNPs and diabetes risk in the DPP population. ${ }^{20}$

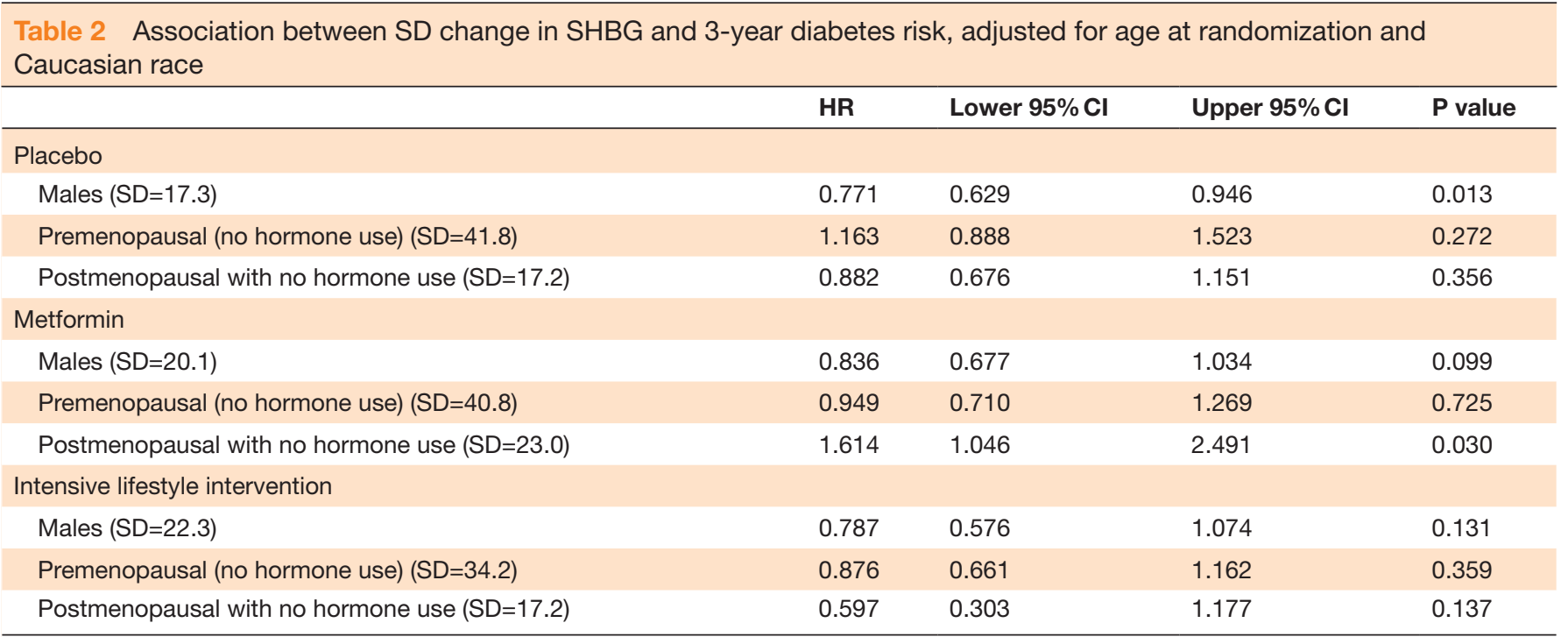

SHBG, sex hormone binding globulin. 


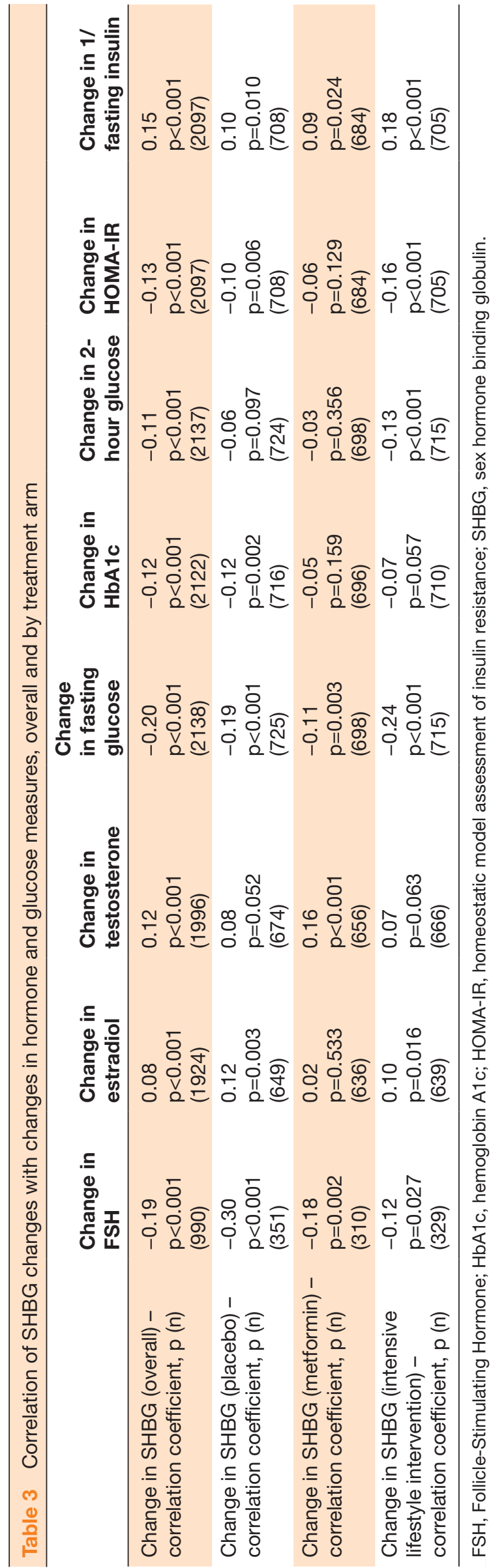

To date, it has not been clear whether low SHBG levels represent a biomarker of metabolic abnormality and diabetes risk or are perhaps somehow contributory and causative of disease. Our data would support that dynamic changes in SHBG reflect the changes in the surrounding metabolic environment. Several studies support this line of thought. First, multiple studies have demonstrated changes in SHBG in response to weight loss, independent of mechanism of weight loss (eg, diet type, exercise, and bariatric surgery). ${ }^{16} 29-32$ In the Sex Hormones and Physical Exercise (SHAPE-2) Study trial, for example, in which overweight inactive women were randomized to diet, exercise or control groups, with a goal of 5-6 kg weight loss over 16 weeks, both the diet and exercise arms achieved weight loss and had significant increases in SHBG, with improvements in sex hormones (increase in free estradiol and decrease in free testosterone) compared with control, yet these effects were attenuated after adjustment for changes in body fat. ${ }^{32}$ In another study, intentional weight loss followed by intentional weight gain in premenopausal women demonstrated both an initial increase in SHBG followed by the reciprocal decrease, with free androgen index and visceral adipose tissue changing in opposite direction to SHBG changes, again speaking to a dynamic change in SHBG in response to decreases in weight and adiposity. ${ }^{33}$ With bariatric surgery, resulting in significant and sustained amounts of weight loss, ${ }^{30} 31$ sustained increases in circulating SHBG have been seen even several years after surgery. ${ }^{31}$

That we did not see an impact of metformin on circulating SHBG is also of interest. This may be because metformin primarily suppresses hepatic glucose production and is not generally regarded as a potent insulin sensitizer. In contrast, intervention studies with insulin sensitizers (thiazolidinediones), have, like for weight loss described previously, demonstrated responsive increases in SHBG. ${ }^{963435}$ In a prospective randomized controlled trial of women with polycystic ovary syndrome, for example, serum SHBG levels were correlated with glucose disposal rate (insulin sensitivity), as assessed by hyperinsulinemic euglycemic clamp, and increased significantly during treatment with pioglitazone, a treatment known to directly modulate insulin sensitivity. Furthermore, the improvement in glucose disposal rate (ie, insulin sensitivity) was directly associated with treatment-associated increases in serum $\mathrm{SHBG}^{9}{ }^{9}$ thus suggesting the effect of insulin sensitization on increasing SHBG. In another study evaluating rosiglitazone (also an insulin sensitizer) on metabolic and ovarian effects in polycystic ovary syndrome (PCOS), an increase in SHBG was demonstrated in response to rosiglitazone treatment for 12 weeks, with higher levels of SHBG seen in those who ovulated on rosiglitazone. Lower circulating insulin levels were also shown, highlighting once again the dynamic changes in SHBG in response to improving insulin sensitivity. ${ }^{34}$ 
Consistent with our findings that SHBG may reflect the metabolic milieu and changes as such, it is of interest to note that the hepatic environment has also been directly implicated in regulating SHBG. Selva et $a l^{36}$ previously showed that monosaccharide (glucose and fructose) induced hepatic lipogenesis reduced SHBG production by downregulating hepatocyte nuclear factor $4 \alpha$ (HNF$4 \alpha$ ) levels, a key transcription factor regulating hepatic expression of SHBG. Supporting this, Winters et $a l^{11}$ evaluated SHBG gene expression in human liver samples and found that SHBG mRNA was a strong predictor of circulating SHBG levels. They described an inverse association between hepatic triglyceride content and SHBG mRNA and serum SHBG, with a suggestion that the low level of SHBG mRNA was largely due to a low level of HNF- $4 \alpha$ mRNA expression in the liver, which is also reduced in insulin resistance. Thus, it is plausible that improvements in insulin sensitivity, as seen with weight loss and therapies that directly modify insulin sensitivity, may have an impact on the liver, which then affects expression of SHBG mRNA and production of SHBG.

In contrast, there is emerging evidence that SHBG may play a causative role in disease and may not merely be an 'innocent bystander'. Sáez-López $e t a l^{\beta 7}$ recently described a significant inverse relation between SHBG mRNA expression and hepatic triglyceride content, as well as levels of acetyl-coenzyme A carboxylase, a key lipogenic enzyme, in liver samples obtained from obese humans with non-alcoholic fatty liver disease undergoing bariatric surgery. Furthermore, the authors found that SHBG overexpression in cultures of human hepatic (HepG2) cells was able to abrogate the increase in multiple hepatic lipogenic enzymes in the liver when triggered under highglucose culture conditions. Although this was studied in fatty liver, it is possible that SHBG is not only a biomarker, but it may independently contribute to the pathogenesis or even protection from metabolic disease.

In order to estimate a causal role of circulating SHBG for type 2 diabetes, Wang et $a l^{38}$ applied quantitative nuclear magnetic resonance metabolomics in three Finnish population-based cohorts to profile circulating lipids and metabolites and their association with SHBG. Higher SHBG levels were associated with a more favorable cardiometabolic risk profile, and SHBG was predictive of future insulin resistance and type 2 diabetes. The observed association of SHBG with type 2 diabetes $(\mathrm{OR}=0.83$ per $1 \mathrm{SD})$ was less than that seen in prospective observational associations by meta-analysis (HR 0.47). These results suggest that circulating SHBG may have a minor direct contributory role in the development of type 2 diabetes but is more likely largely reflective of other factors.

There are several strengths and limitations to these analyses. First, a prospective, controlled evaluation of changes in SHBG by sex and menopausal status in the very well characterized DPP population provided the opportunity to directly assess whether the study interventions affected SHBG and what other factors contributed to those changes. In addition, our sample size is commensurate with other studies in the literature ${ }^{1278}$ for the relevant constituent grouping to have provided meaningful analyses and comparative results. However, given the predefined criteria of the DPP to identify those individuals already at high risk of development of diabetes based on glucose and weight measures, we may have been limited in seeing additional impact of SHBG on risk. Furthermore, SHBG and sex hormone measurements were conducted on samples that were not timed to the endogenous hormonal cycle, and hormone use was assessed by self-report. Randomization may help to minimize potential influence of this and other unanticipated factors that may regulate SHBG.

In summary, in the DPP, ILS was consistently associated with changes in circulating SHBG levels compared with placebo or metformin, specifically increased levels in postmenopausal women, and attenuation of decrease in men and premenopausal women. The effects of ILS on SHBG seemed largely due to changes in adiposity but may also be influenced by other changes (eg, glucose measures and sex steroids). The observed changes in SHBG related to the interventions did not consistently translate to changes in diabetes risk.

\section{Author affiliations}

${ }^{1}$ MedStar Health Research Institute, Hyattsville, Maryland, USA

${ }^{2}$ Department of Medicine, Brigham and Women's Hospital, Harvard Medical School, Boston, Massachusetts, USA

${ }^{3}$ The Biostatistics Center, The George Washington University Milken Institute of Public Health, Rockville, Maryland, USA

${ }^{4}$ Department of Medicine, University of Colorado Anschutz Medical Campus, Aurora, Colorado, USA

${ }^{5}$ Departments of Medicine, Obstetrics \& Gynecology, and Epidemiology, University of Michigan, Ann Arbor, Michigan, USA

${ }^{6}$ Division of Endocrinology, Johns Hopkins University School of Medicine, Baltimore, Maryland, USA

${ }^{7}$ Joslin Diabetes Center, Boston, Massachusetts, USA

${ }^{8}$ Indiana University School of Medicine, Indianapolis, Indiana, USA

Acknowledgements The Research Group gratefully acknowledges the commitment and dedication of the participants of the Diabetes Prevention Program (DPP) and the Diabetes Prevention Program Outcomes Study (DPPOS). During the DPP and DPPOS, the National Institute of Diabetes and Digestive and Kidney Diseases (NIDDK) of the National Institutes of Health provided funding to the clinical centers and the Coordinating Center for the design and conduct of the study, and collection, management, analysis, and interpretation of the data (U01 DK048489). The Southwestern American Indian Centers were supported directly by the NIDDK, including its Intramural Research Program, and the Indian Health Service. The General Clinical Research Center Program, National Center for Research Resources, and the Department of Veterans Affairs supported data collection at many of the clinical centers. Funding was also provided by the National Institute of Child Health and Human Development, the National Institute on Aging, the National Eye Institute, the National Heart Lung and Blood Institute, the National Cancer Institute, the Office of Research on Women's Health, the National Institute on Minority Health and Health Disparities, the Centers for Disease Control and Prevention, and the American Diabetes Association. Bristol-Myers Squibb and Parke-Davis provided additional funding and material support during the DPP, Lipha (MerckSante) provided medication and LifeScan Inc donated materials during the DPP and DPPOS. This research was also supported, in part, by the intramural research program of the NIDDK. LifeScan Inc, Health 0 Meter, Hoechst Marion Roussel, Inc, Merck-Medco Managed Care, Inc, Merck and Co, Nike Sports Marketing, Slim Fast Foods $\mathrm{C}_{0}$, and Quaker Oats $\mathrm{Co}$ donated materials, equipment, or medicines for concomitant conditions. Endoceutics, Inc, McKesson BioServices Corp, Matthews Media Group, Inc, and the Henry M. Jackson Foundation provided support services 
under subcontract with the Coordinating Center. Dr Fernand Labrie, through Endoceutics Inc, provided hormone measurements on a contractual basis. The sponsor of this study was represented on the Steering Committee and played a part in study design, how the study was done, and publication. The funding agency was not represented on the writing group, although all members of the Steering Committee had input into the report's contents. All authors in the writing group had access to all data. A complete list of centers, investigators, and staff can be found in the Appendix.

Contributors The manuscript was conceived by VRA and KJM, with manuscript questions and analytic plan designed by VRA, CAC, CK, SLE and KJM. VRA wrote the manuscript, interpreted the data, critically reviewed and revised the manuscript. CAC contributed to writing, data analysis, data interpretation, critical review and revision. SLE contributed to data analysis, data interpretation, critical review and revision. LP, CK, SHG, EH and KJM contributed to data interpretation, critical review and revision. All authors had access to the data and all authors agreed to submit the final manuscript. At the time of publication KJM was a full-time employee of Eli Lilly and Company. However, prior to employment at Eli Lilly and Company, KJM served as Principal Investigator for this NIH funded study. As such, data collection occurred prior to and independent of this employment and therefore, data analysis and preparation of the manuscript were independent of Eli Lilly and Company.

Funding The DPP/DPPOS was funded by National Institute of Diabetes and Digestive and Kidney Disease, award U01 DK048489.

Disclaimer The opinions expressed are those of the investigators and do not necessarily reflect the views of the funding agencies. The funders had no role in study design, data collection and analysis, decision to publish, or preparation of the manuscript.

Competing interests None declared.

Patient consent for publication Not required.

Ethics approval All 27 DPP clinical study centers, as well as the DPP Coordinating Center, had institutional review board approvals.

Provenance and peer review Not commissioned; externally peer reviewed.

Data availability statement Data may be obtained from a third party and are not publicly available. In accordance with the NIH Public Access Policy, we continue to provide all manuscripts to PubMed Central including this manuscript. DPP/DPPOS has provided the protocols and lifestyle and medication intervention manuals to the public through its public website (https://www.dppos.org). The DPPOS abides by the NIDDK data sharing policy and implementation guidance as required by the NIH/NIDDK (https://www.niddkrepository.org/studies/dppos/)

Supplemental material This content has been supplied by the author(s). It has not been vetted by BMJ Publishing Group Limited (BMJ) and may not have been peer-reviewed. Any opinions or recommendations discussed are solely those of the author(s) and are not endorsed by BMJ. BMJ disclaims all liability and responsibility arising from any reliance placed on the content. Where the content includes any translated material, BMJ does not warrant the accuracy and reliability of the translations (including but not limited to local regulations, clinical guidelines, terminology, drug names and drug dosages), and is not responsible for any error and/or omissions arising from translation and adaptation or otherwise.

Open access This is an open access article distributed in accordance with the Creative Commons Attribution Non Commercial (CC BY-NC 4.0) license, which permits others to distribute, remix, adapt, build upon this work non-commercially, and license their derivative works on different terms, provided the original work is properly cited, appropriate credit is given, any changes made indicated, and the use is non-commercial. See: http://creativecommons.org/licenses/by-nc/4.0/.

ORCID IDs

Sharon L Edelstein http://orcid.org/0000-0001-8457-4404

Sherita H Golden http://orcid.org/0000-0001-8854-4026

\section{REFERENCES}

1 Lakshman KM, Bhasin S, Araujo AB. Sex hormone-binding globulin as an independent predictor of incident type 2 diabetes mellitus in men. J Gerontol A Biol Sci Med Sci 2010;65:503-9.

2 Ding EL, Song Y, Manson JE, et al. Sex hormone-binding globulin and risk of type 2 diabetes in women and men. $N$ Engl $J$ Med 2009;361:1152-63.

3 Muka T, Nano J, Jaspers L, et al. Associations of steroid sex hormones and sex hormone-binding globulin with the risk of type 2 diabetes in women: a population-based cohort study and metaanalysis. Diabetes 2017;66:577-86.

$4 \mathrm{Hu} \mathrm{J}$, Zhang A, Yang S, et al. Combined effects of sex hormonebinding globulin and sex hormones on risk of incident type 2 diabetes. J Diabetes 2016;8:508-15.

$5 \mathrm{Kim} \mathrm{C}$, Halter JB. Endogenous sex hormones, metabolic syndrome, and diabetes in men and women. Curr Cardiol Rep 2014;16:467.

6 Ding EL, Song Y, Malik VS, et al. Sex differences of endogenous sex hormones and risk of type 2 diabetes: a systematic review and meta-analysis. JAMA 2006;295:1288-99.

7 Chen BH, Brennan K, Goto A, et al. Sex hormone-binding globulin and risk of clinical diabetes in American black, Hispanic, and Asian/Pacific Islander postmenopausal women. Clin Chem 2012;58:1457-66.

8 Kalyani RR, Franco M, Dobs AS, et al. The association of endogenous sex hormones, adiposity, and insulin resistance with incident diabetes in postmenopausal women. J Clin Endocrinol Metab 2009;94:4127-35.

9 Aroda VR, Ciaraldi TP, Burke P, et al. Metabolic and hormonal changes induced by pioglitazone in polycystic ovary syndrome: a randomized, placebo-controlled clinical trial. J Clin Endocrinol Metab 2009;94:469-76.

10 Akin F, Bastemir M, Alkis E, et al. Shbg levels correlate with insulin resistance in postmenopausal women. Eur J Intern Med 2009;20:162-7.

11 Winters SJ, Gogineni J, Karegar M, et al. Sex hormone-binding globulin gene expression and insulin resistance. J Clin Endocrinol Metab 2014;99:E2780-8.

12 Arias-Santiago S, Gutiérrez-Salmerón MT, Buendía-Eisman A, et al. Sex hormone-binding globulin and risk of hyperglycemia in patients with androgenetic alopecia. J Am Acad Dermatol 2011;65:48-53.

13 Colangelo LA, Ouyang P, Liu K, et al. Association of endogenous sex hormones with diabetes and impaired fasting glucose in men: multiethnic study of atherosclerosis. Diabetes Care 2009;32:1049-51.

14 Barba M, Schünemann HJ, Sperati F, et al. The effects of metformin on endogenous androgens and SHBG in women: a systematic review and meta-analysis. Clin Endocrinol 2009;70:661-70.

15 Armamento-Villareal R, Aguirre LE, Qualls C, et al. Effect of lifestyle intervention on the hormonal profile of frail, obese older men. J Nutr Health Aging 2016;20:334-40.

16 Morisset A-S, Blouin K, Tchernof A. Impact of diet and adiposity on circulating levels of sex hormone-binding globulin and androgens. Nutr Rev 2008;66:506-16.

17 Mingrone G, Greco AV, Giancaterini A, et al. Sex hormone-binding globulin levels and cardiovascular risk factors in morbidly obese subjects before and after weight reduction induced by diet or malabsorptive surgery. Atherosclerosis 2002;161:455-62.

18 Knowler WC, Barrett-Connor E, Fowler SE, et al. Reduction in the incidence of type 2 diabetes with lifestyle intervention or metformin. N Engl J Med 2002;346:393-403.

19 Kim C, Kong S, Laughlin GA, et al. Endogenous sex hormone changes in postmenopausal women in the diabetes prevention program. J Clin Endocrinol Metab 2012;97:2853-61.

20 Mather KJ, Kim C, Christophi CA, et al. Steroid sex hormones, sex hormone-binding globulin, and diabetes incidence in the diabetes prevention program. J Clin Endocrinol Metab 2015;100:3778-86.

21 Diabetes Prevention Program Research Group, Knowler WC, Fowler SE, et al. 10-Year follow-up of diabetes incidence and weight loss in the diabetes prevention program outcomes study. Lancet 2009;374:1677-86.

22 Singh B, Saxena A. Surrogate markers of insulin resistance: a review. World J Diabetes 2010;1:36-47.

$23 \mathrm{Ke} \mathrm{Y,} \mathrm{Bertin} \mathrm{J,} \mathrm{Gonthier} \mathrm{R,} \mathrm{et} \mathrm{al.} \mathrm{A} \mathrm{sensitive,} \mathrm{simple} \mathrm{and} \mathrm{robust}$ LC-MS/MS method for the simultaneous quantification of seven androgen- and estrogen-related steroids in postmenopausal serum. $J$ Steroid Biochem Mol Biol 2014;144 Pt B:523-34.

24 Labrie F, Bélanger $A$, Bélanger $P$, et al. Androgen glucuronides, instead of testosterone, as the new markers of androgenic activity in women. J Steroid Biochem Mol Biol 2006;99:182-8.

25 Wallace IR, McKinley MC, Bell PM, et al. Sex hormone binding globulin and insulin resistance. Clin Endocrinol 2013;78:321-9.

26 Deswal R, Yadav A, Dang AS. Sex hormone binding globulin - an important biomarker for predicting PCOS risk: A systematic review and meta-analysis. Syst Biol Reprod Med 2018;64:12-24.

27 Jaruvongvanich V, Sanguankeo A, Riangwiwat T, et al. Testosterone, sex hormone-binding globulin and nonalcoholic fatty liver disease: a systematic review and meta-analysis. Ann Hepatol 2017;16:382-94.

28 Kavanagh K, Espeland MA, Sutton-Tyrrell K, et al. Liver fat and SHBG affect insulin resistance in midlife women: The Study of Women's Health Across the Nation (SWAN). Obesity 2013:21:1031-8. 
29 Moran LJ, Brinkworth GD, Martin S, et al. Long-Term effects of a randomised controlled trial comparing high protein or high carbohydrate weight loss diets on testosterone, SHBG, erectile and urinary function in overweight and obese men. PLoS One 2016;11:e0161297.

30 Lee Y, Dang JT, Switzer N, et al. Impact of bariatric surgery on male sex hormones and sperm quality: a systematic review and metaanalysis. Obes Surg 2019;29:334-46.

31 Sarwer DB, Spitzer JC, Wadden TA, et al. Sexual functioning and sex hormones in men who underwent bariatric surgery. Surg Obes Relat Dis 2015;11:643-51.

32 van Gemert WAM, Schuit AJ, van der Palen J, et al. Effect of weight loss, with or without exercise, on body composition and sex hormones in postmenopausal women: the SHAPE-2 trial. Breast Cancer Res 2015;17:120.

33 Aubuchon M, Liu Y, Petroski GF, et al. The impact of supervised weight loss and intentional weight regain on sex hormone binding globulin and testosterone in premenopausal women. Syst Biol Reprod Med 2016;62:283-9.
34 Cataldo NA, Abbasi F, McLaughlin TL, et al. Metabolic and ovarian effects of rosiglitazone treatment for 12 weeks in insulinresistant women with polycystic ovary syndrome. Hum Reprod 2006;21:109-20.

35 Kapoor D, Channer KS, Jones TH. Rosiglitazone increases bioactive testosterone and reduces waist circumference in hypogonadal men with type 2 diabetes. Diab Vasc Dis Res 2008;5:135-7.

36 Selva DM, Hogeveen KN, Innis SM, et al. Monosaccharide-induced lipogenesis regulates the human hepatic sex hormone-binding globulin gene. J Clin Invest 2007;117:3979-87.

37 Sáez-López C, Salcedo-Allende MT, Hernandez C, et al. Sex hormone-binding globulin expression correlates with acetylcoenzyme A carboxylase and triglyceride content in human liver. $J$ Clin Endocrinol Metab 2019;104:1500-7.

38 Wang Q, Kangas AJ, Soininen P, et al. Sex hormone-binding globulin associations with circulating lipids and metabolites and the risk for type 2 diabetes: observational and causal effect estimates. Int $J$ Epidemiol 2015;44:623-37. 